\title{
Rotinas de Cuidados Pós-Anestésicos de Anestesiologistas Brasileiros *
}

\section{Postanesthetic Routines of Brazilian Anesthesiologists}

Getúlio Rodrigues de Oliveira Filho, TSA ${ }^{1}$

\author{
RESUMO \\ Oliveira $F^{\circ}$ GR - Rotinas de Cuidados Pós-Anestésicos de \\ Anestesiologistas Brasileiros
}

\begin{abstract}
JUSTIFICATIVA E OBJETIVOS: Não existem dados sobre a disponibilidade de equipamentos nas Salas de Recuperação Pós-Anestésica (SRPA), as rotinas de monitorização e os critérios de alta pós-anestésica utilizados pelos anestesiologistas brasileiros. Este estudo visou obter estas informações.
\end{abstract}

MÉTODO: Foi pesquisada uma amostra aleatória de 1123 anestesiologistas. Os dados foram analisados por tabulação simples e cruzada e por regressão logística.

RESULTADOS: Foram analisados 271 questionários (26,59\%). O estudo revelou que a maioria dos anestesiologistas atua em instituições que possuem SRPA equipadas com dispositivos de reanimação, fontes de oxigênio, aspiradores, cardioscópios, oxímetros de pulso e que mantêm anestesiologista de plantão. A existência de monitores de função neuromuscular e dispositivos de aquecimento ativo é menos freqüente. Em ordem decrescente de freqüência, são monitorizados rotineiramente a pressão arterial, a freqüência cardíaca, a $\mathrm{SpO}_{2}$, o nível de consciência, a patência das vias aéreas, a freqüência respiratória, as náuseas e vômitos, a dor pós-operatória e a força muscular. A oxigenioterapia, os antieméticos e o tratamento de calafrios pós-operatórios são administrados rotineiramente ou a pacientes selecionados pela maioria dos anestesiologistas. A maioria dos anestesiologistas registra os dados pós-anestésicos.

CONCLUSÕES: Este estudo sugere que os anestesiologistas brasileiros preocupam-se com a segurança dos pacientes no período pós-anestésico imediato, conforme atestam a elevada disponibilidade de equipamentos e os percentuais expressivos de monitorização de parâmetros isolados. Algumas rotinas de monitorização precisam ser implementadas de acordo com as novas diretrizes de cuidados pós-anestésicos e os novos critérios de alta para pacientes ambulatoriais.

Unitermos: ANESTESIOLOGIA: pesquisa; ANESTESIOLOGISTA; RECUPERAÇÃO PÓS-ANESTÉSICA: critérios de alta, sala

\footnotetext{
* Recebido do (Received from) CET/SBA Integrado de Anestesiologia da

SES-SC, Florianópolis, SC

1. Instrutor; Co-responsável do CET/SBA
}

Endereço para Correspondência (Correspondence to)

Dr. Getúlio Rodrigues de Oliveira Filho

Rua Luiz Delfino 111/902

88015-360 Florianópolis - SC

E-mail: grof@th.com.br

Apresentado (Submitted) em 24 de setembro de 2002

Aceito (Accepted) para publicação em 16 de dezembro de 2003

(c) Sociedade Brasileira de Anestesiologia, 2003

\author{
SUMMARY \\ Oliveira $F^{\circ} \mathrm{GR}$ - Postanesthetic Routines of Brazilian Anesthe- \\ siologists
}

BACKGROUND AND OBJECTIVES: There are no data on Postanesthetic Care Units (PACU) equipment, monitoring routines and discharge criteria adopted by Brazilian anesthesiologists. This study aimed at obtaining such data.

METHODS: A nationwide survey was conducted with a random sample of 1123 anesthesiologists. Data were analyzed by simple and cross tabulation and logistic regression.

RESULTS: Two hundred seventy-one questionnaires (26.59\%) were analyzed. The study showed that most anesthesiologists work in institutions with PACUs equipped with resuscitation equipment, oxygen sources, aspirators, cardioscopes, pulse oximeters and with an anesthesiologist on duty. Neuromuscular function monitors and active heating devices are less frequent. In a descending order of frequency, the following parameters are routinely monitored: blood pressure, heart rate, $\mathrm{SpO}_{2}$, consciousness level, airway patency, respiratory rate, nausea and vomiting, postoperative pain and muscle strength. Oxygen therapy, anti-emetics and postanesthetic shivering control are prescribed on a routine or selective basis by most anesthesiologists who also record postoperative data.

CONCLUSIONS: This study suggests that Brazilian anesthesiologists are concerned about patients' safety during postanesthetic recovery, as shown by high equipment availability in the PACU and expressive routine monitoring ratios of isolated parameters. Some monitoring routines should be implemented to comply with new guidelines on postanesthetic care and new discharge criteria for outpatient procedures.

Key Words: ANESTHESIOLOGIST; ANESTHESIOLOGY, research; POSTANESTHETIC RECOVERY: discharge criteria, room

\section{INTRODUÇÃO}

$\mathrm{O}$ cuidados pós-anestésicos compreendem as atividades de monitorização e tratamento utilizadas para o manuseio do paciente após um procedimento anestésico-cirúrgico, através das diversas fases da recuperação anestésica. Na maior parte das vezes, a recuperação inicial da anestesia (estágio I) ocorre ainda na sala cirúrgica, quando o objetivo primordial é o restabelecimento da atividade e dos reflexos respiratórios, da estabilidade cardiovascular e da força muscular. Em uma segunda fase (estágio II), na Sala de Recuperação Pós-Anestésica (SRPA), o paciente é observado sob monitorização clínica e instrumental, quanto aos mesmos parâmetros, adicionados do nível de consciência. Pacientes submetidos a bloqueios neuroaxiais permanecem na SRPA até que o bloqueio simpático se dissipe. É nessa fase que a dor pós-operatória, a ocorrência de náuseas e vômitos, de calafrios, de retenção urinária, de sangramentos anormais adquire um papel fun- 
damental, porque seu tratamento implica maior conforto e segurança para o paciente. Pacientes submetidos a cirurgias em regime ambulatorial passam por uma terceira fase da recuperação anestésica (estágio III) ainda na SRPA ou em ambiente próprio de uma unidade de cirurgia ambulatorial, onde são asseguradas a capacidades de deambulação, de micção espontânea, de tolerar líquidos por via oral e de ter a dor, as náuseas ou vômitos controlados por medicação oral ${ }^{1}$.

AAmerican Society of Anesthesiologists (ASA) recentemente publicou o relatório de um grupo de trabalho designado para a elaboração de diretrizes práticas para o cuidado pós-operatório ${ }^{2}$, cujas recomendações podem ser assim resumidas:

1. A SRPA deve possuir equipamentos para reanimação cardiorrespiratória;

2. Deve haver um anestesiologista à disposição para o tratamento de intercorrências na SRPA;

3. Devem ser monitorizados de rotina, na SRPA os seguintes parâmetros: a freqüência respiratória, a patência das vias aéreas, a saturação periférica da oxihemoglobina $\left(\mathrm{SpO}_{2}\right)$, a freqüência cardíaca, a pressão arterial, a função neuromuscular por exame clínico, o estado mental, a dor e as náuseas e vômitos pós-operatórios;

4. Em pacientes selecionados, por fatores de risco ou pelo quadro clínico, devem ser monitorizados o traçado eletrocardiográfico, por cardioscopia, a função neuromuscular por estimulador de nervo periférico, a temperatura, o débito urinário, a capacidade de micção, o débito de drenos e sondas e/ou o sangramento pós-operatório;

5. Os antieméticos devem ser utilizados para profilaxia de náuseas e vômitos em pacientes selecionados, segundo fatores de risco e devem ser utilizados de rotina para seu tratamento;

6. A oxigenioterapia deve ser utilizada em pacientes selecionados por fatores de risco de hipoxemia, ou para o seu tratamento;

7. Os dispositivos de aquecimento ativo (ar forçado) devem ser utilizados para a profilaxia e tratamento de hipotermia;

8. Os calafrios pós-operatórios devem ser tratados rotineiramente. A meperidina é a droga de escolha.

9. As exigências para que os pacientes urinem ou que ingiram líquidos antes de deixarem o hospital devem ser aplicadas somente a pacientes portadores de fatores de risco para retenção urinária ou náuseas e vômitos, respectivamente;

10. O período de observação pós-anestésica deve ser individualizado de acordo com a magnitude do risco de depressão cardiorrespiratória, de tal forma que um período mínimo de permanência na SRPA não é justificado;

11. Os pacientes ambulatoriais somente devem deixar o hospital acompanhados por adulto responsável e após receberem, os pacientes e seus acompanhantes, instruções, por escrito, quanto aos cuidados pós-anestésicos e a formas de contatos com o hospital, em caso de complicações.
No Brasil, a existência obrigatória de SRPA em hospitais foi determinada pela Portaria 400 do Ministério da Saúde, em 1977. A Resolução CFM n ${ }^{\circ} 1363 / 93$, que trata da segurança em anestesia, estabelece, no artigo VI, que "todo o paciente após a cirurgia deverá ser removido para a sala de recuperação pós-anestésica" e no artigo VIII, que "os critérios de alta do paciente no período de recuperação pós-anestésica são de responsabilidade intransferível do anestesista". A Resolução CFM n ${ }^{\circ}$ 1409/94, que trata da normatização da cirurgia ambulatorial, prevê, em seu artigo III, que as condições para alta do paciente ambulatorial são: orientação no tempo e espaço, estabilidade de sinais vitais há, pelo menos, 60 minutos, ausência de náuseas ou vômitos, ausência de dificuldade respiratória, capacidade de ingerir líquidos, capacidade de locomoção como a pré-operatória, sangramento operatório mínimo ou ausente, ausência de dor de grande intensidade e de sinais de retenção urinária. Prevê também que, ao paciente e seu acompanhante maior e responsável, sejam dadas, verbalmente e por escrito, instruções relativas aos cuidados pós-anestésicos e pós-operatórios, bem como a determinação da unidade para atendimento das eventuais intercorrências.

Este estudo teve por objetivos primários determinar:

1. Qual a disponibilidade de equipamentos nas SRPA das instituições onde os participantes desenvolvem suas atividades principais;

2. Como os participantes monitorizam os parâmetros indicados como rotineiros e os indicados para pacientes selecionados;

3. Em que circunstâncias são utilizados a oxigenioterapia, a profilaxia farmacológica de náuseas e vômitos e o tratamento dos calafrios pós-operatórios;

4. Como são utilizados os critérios de alta propostos na literatura e na legislação vigente para pacientes internados e ambulatoriais submetidos a anestesias gerais ou neuroaxiais;

5. Como são cumpridos os requisitos médico-legais referentes à recuperação pós-anestésica.

Secundariamente, o estudo visou estudar os efeitos da região geográfica, das características das instituições (regime jurídico, caráter universitário e existência de Programa de Residência Médica em Anestesiologia (PRM) e existência de formulário para registro do escore de Aldrete-Kroulik e da qualificação profissional (experiência profissional e Título Superior em Anestesiologia (TSA)) sobre a disponibilidade de recursos humanos e materiais, as rotinas de monitorização e tratamento na SRPA e o cumprimento das exigências médico-legais.

\section{MÉTODO}

Foram obtidos 2246 endereços eletrônicos de membros da Sociedade Brasileira de Anestesiologia (SBA), através de consulta ao portal da SBA (www.sba.com.br) na rede mundial de computadores (Internet). Para o cálculo do tamanho da 
amostra, foi estimado, com base em outro estudo ${ }^{2}$, que pelo menos $80 \%$ dos participantes considerariam rotineira a monitorização da freqüência respiratória, da patência das vias aéreas, da $\mathrm{SpO}_{2}$, da freqüência cardíaca, da pressão arterial, da força muscular, do nível de consciência, da intensidade da dor pós-operatória e das náuseas e vômitos. Para um erro aceitável de $5 \%$ e limites de confiança de $95 \%$, o tamanho calculado da amostra foi igual a 256. Tomando uma taxa de retorno estimada em $25 \%{ }^{2-4}$, foi estabelecido em 1024 o número mínimo de anestesiologistas a serem consultados. Foram escolhidos 1123 anestesiologistas, por amostragem probabilística aleatória simples, com probabilidade de cada endereço da lista ser escolhido igual a 1:2 ${ }^{3-4}$.

A mensagem, enviada por correio eletrônico, continha uma introdução que descrevia o objetivo da pesquisa, seguida do questionário. As questões foram apresentadas em quatro blocos.

O bloco I contemplou os seguintes itens:

1. Região de atuação - norte, nordeste, centro-oeste, sudeste ou sul;

2. Experiência profissional - médico residente, anestesiologista há menos de cinco anos ou anestesiologista há cinco anos ou mais;

3. Título Superior em Anestesiologia - sim ou não;

4. Principal característica da instituição - pública, universitária ou não, ou privada, universitária ou não;

5. Presença de Programa de Residência Médica em Anestesiologia na instituição de atuação principal - sim ou não;

6. Localização da SRPA dentro do Centro Cirúrgico, existência de centro cirúrgico exclusivo para cirurgias ambulatoriais, de sala de recuperação exclusiva para pacientes ambulatoriais, de anestesiologista de plantão nas 24 horas na instituição, de anestesiologista de plantão nas 24 horas exclusivamente para a SRPA, de equipamentos de reanimação na SRPA, incluindo desfibrilador - sim ou não;

7. Número de salas do Centro Cirúrgico, da SRPA, da Unidade de Cirurgia Ambulatorial e da Sala de Recuperação da Unidade de Cirurgia Ambulatorial.

No bloco II, foi solicitado aos participantes que assinalassem a situação (rotina, em pacientes selecionados ou nunca) em que cada um dos seguintes parâmetros é monitorizado, na SRPA, na sua prática: freqüência respiratória, patência das vias aéreas, saturação periférica da oxihemoglobina $\left(\mathrm{SpO}_{2}\right)$, freqüência cardíaca, pressão arterial, traçado eletrocardiográfico com cardioscópio, força muscular por exame clínico, força muscular por estimulador de nervo periférico, estado mental ou nível de consciência, temperatura, intensidade da dor pós-operatória, freqüência e intensidade de náuseas e vômitos, débito urinário, drenagem e sangramento. Neste bloco ainda foi questionado se os parâmetros são registrados no prontuário e se existe formulário próprio para o registro dos escores de Aldrete-Kroulik disponível nas instituições onde atuam.
No bloco III foi solicitado aos participantes que classificassem a utilização e a disponibilidade de cardioscópio, oxímetro de pulso, monitor de função neuromuscular, aspirador, fonte de oxigênio e dispositivo de aquecimento ativo para uso na SRPA, segundo as categorias: utilizado/disponível para rotina, utilizado/disponível para pacientes selecionados ou não utilizado/não disponível.

No bloco IV, foi solicitado aos participantes que marcassem uma das alternativas (utilizo como rotina, utilizo em pacientes selecionados ou nunca utilizo) que refletisse sua posição frente às seguintes condutas, na SRPA:

1. Uso de oxigenioterapia;

2. Uso de antieméticos profiláticos;

3. Tratamento farmacológico com meperidina e oxigênio de calafrios pós-anestésicos;

4. Micção mandatória antes da alta de pacientes ambulatoriais;

5. Acompanhante responsável para pacientes ambulatoriais;

6. Tempo de permanência mínimo na SRPA preestabelecido;

7. Escore de Aldrete-Kroulik igual ou superior a nove antes da alta da SRPA;

8. Regressão do nível superior do bloqueio sensitivo a, pelo menos, L1, antes da alta de pacientes internados submetidos a bloqueios neuroaxiais;

9. Capacidade de movimentação dos membros inferiores antes da alta de pacientes internados submetidos a bloqueios neuroaxiais;

10. Orientações escritas pós-anestésicas para pacientes ambulatoriais;

11. Alta da SRPA delegada a enfermeiro ou técnico em enfermagem treinado.

O questionário foi encerrado por uma afirmação de concordância com a publicação dos dados fornecidos sob a forma de artigo científico, mantido o sigilo quanto à origem que deveria ser assinalada, caso positivo e por instruções sobre como responder à mensagem.

Aanálise estatística foi composta de duas etapas. A primeira, visando o cumprimento dos objetivos primários, constou de tabulação simples das respostas e cálculos dos respectivos percentuais. Na etapa seguinte, foi conduzida a análise exploratória dos dados, com o fim de cumprir os objetivos secundários do estudo. Cada item do questionário (variável dependente) foi categorizado, segundo as seguintes variáveis independentes:

a) Região geográfica de atuação (norte/nordeste, centro-oeste, sul e sudeste);

b) Qualificação profissional do participante: nível de experiência profissional (Médico em Especialização, Anestesiologista com experiência menor do que 5 anos, anestesiologista com cinco anos ou mais de experiência) e Título Superior em Anestesiologia (portador ou não); 
c) Características das instituições de atuação dos participantes: caráter universitário ou não, regime jurídico público ou privado, presença ou ausência de PRM, existência ou inexistência de formulário para registro do escore de Aldrete-Kroulik.

As variáveis categóricas foram submetidos a testes de Qui-quadrado e as ordinais a testes de Mann-Whitney ou Kruskal-Wallis, seguidos de regressão logística retrógrada. O nível de significância foi estabelecido em $5 \%$.

\section{RESULTADOS}

Dos 1123 questionários enviados, 104 foram devolvidos sob a alegação de endereço eletrônico inexistente. Dos 1019 aceitos, 294 foram respondidos, correspondendo a uma taxa de resposta de $28,85 \%$. Dos que responderam ao questionário, 23 não concordaram com a utilização dos dados fornecidos para fins de publicação, de forma que a análise dos resultados foi realizada com 271 participantes $(26,59 \%$ dos questionários enviados).

As tabulações simples das respostas são mostradas nas tabelas I a VIII.

A análise multivariada identificou as variáveis independentes que se associaram a maiores (razões de chances maiores que 1) ou menores (razões de chances menores que 1) probabilidades de disponibilidade de recursos humanos e materiais, de práticas de monitorização e de cumprimento de exigência médico-legais. As associações significativas são descritas a seguir. Os valores apresentados entre colchetes referem-se às razões de chances e aos seus respectivos intervalos de $95 \%$ de confiança, entre parênteses:

Tabela I - Características Gerais da Amostra

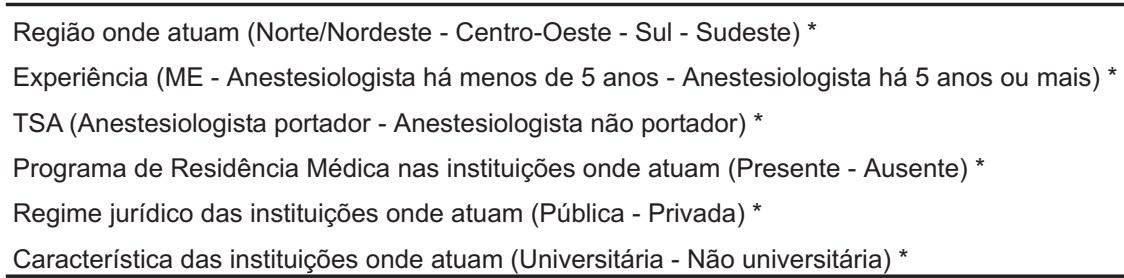

* = Os valores representam o número de respostas em cada categoria com os respectivos percentuais, entre parênteses

Tabela II - Características das Instituições onde Atuam os Participantes

\begin{tabular}{|c|c|c|c|}
\hline Características & Sim & Não & Não respondeu \\
\hline SRPA localizada dentro do Centro Cirúrgico * & $254(93,72)$ & $16(5,9)$ & $1(0,36)$ \\
\hline Centro Cirúrgico exclusivo para cirurgias ambulatoriais * & $95(35,05)$ & $176(64,94)$ & $0(0)$ \\
\hline Sala de recuperação exclusiva para pacientes ambulatoriais * & $74(29,30)$ & $197(72,69)$ & $0(0)$ \\
\hline Anestesiologista de plantão ininterrupto * & $206(76,01)$ & $63(23,24)$ & $2(0,73)$ \\
\hline Anestesiologista de plantão exclusivo para a SRPA * & $40(17,76)$ & $228(84,13)$ & $3(1,10)$ \\
\hline Número de salas do Centro Cirúrgico & 7 & $0-33$ & $5-10$ \\
\hline Número de leitos da SRPA & 8 & $0-40$ & $5-10$ \\
\hline Razão entre o número de leitos da SRPAe o número de salas do Centro Cirúrgico & 1 & $0-3,07$ & $0,61-1,25$ \\
\hline Número de salas do Centro Cirúrgico Ambulatorial (CCA) & 3 & $1-16$ & $2-4$ \\
\hline Número de leitos da Sala de Recuperação Ambulatorial (SRA) & 4 & $1-25$ & $3-6$ \\
\hline Razão entre o número de leitos da SRA e o número de salas do CCA & 0,5 & $0,2-5$ & $12,5 ?$ \\
\hline
\end{tabular}

* = Os valores representam o número de respostas em cada categoria com os respectivos percentuais, entre parênteses

Tabela III - Disponibilidade de Equipamentos nas SRPA das Instituições onde Atuam os Participantes

\begin{tabular}{|c|c|c|c|}
\hline & Disponível & Não disponível & IC95\% \\
\hline Equipamento de reanimação cardiorrespiratória, incluindo desfibrilador * & $235(86,71)$ & $36(13,28)$ & $\pm 0,17 \%$ \\
\hline Fonte de oxigênio * & $267(98,52)$ & $4(1,47)$ & $\pm 0,02 \%$ \\
\hline Aspirador * & $270(99,63)$ & $1(0,36)$ & $\pm 0 \%$ \\
\hline Cardioscópio * & $264(97,41)$ & $7(2,58)$ & $\pm 0,03 \%$ \\
\hline Oxímetro de pulso * & $268(98,89)$ & $3(1,10)$ & $\pm 0,01 \%$ \\
\hline
\end{tabular}

* = Os valores representam o número de respostas em cada categoria com os respectivos percentuais, entre parênteses 
Tabela IV - Rotinas de Monitorização na SRPA dos Parâmetros Considerados Essenciais

\begin{tabular}{|c|c|c|c|c|c|}
\hline Parâmetros & Rotina & Pacientes Selecionados & Nunca & Sem Resposta & $\mathrm{IC} 95 \%$ \\
\hline Pressão arterial * & $259(95,57)$ & $11(4,05)$ & $1(0,36)$ & $0(0)$ & $\pm 0,06 \%$ \\
\hline Freqüência cardíaca * & $257(94,83)$ & $10(3,69)$ & $0(0)$ & $4(1,47)$ & $\pm 0,07 \%$ \\
\hline $\mathrm{SpO}_{2}$ * & $226(83,39)$ & $43(15,86)$ & $0(0)$ & $2(0,73)$ & $\pm 0,20 \%$ \\
\hline Nível de consciência/estado mental * & $225(83,02)$ & $36(13,28)$ & $9(3,32)$ & $1(0,36)$ & $\pm 0,20 \%$ \\
\hline Patência das vias aéreas * & $193(71,21)$ & $58(21,40)$ & $15(5,53)$ & $5(1,84)$ & $\pm 0,30 \%$ \\
\hline Intensidade de náuseas e vômitos * & $174(64,20)$ & $72(26,56)$ & $22(8,11)$ & $3(1,10)$ & $\pm 0,33 \%$ \\
\hline Intensidade da dor pós-operatória * & $172(63,46)$ & $69(25,46)$ & $26(9,59)$ & $4(1,47)$ & $\pm 0,34 \%$ \\
\hline Força muscular (exame clínico) * & $92(33,94)$ & $143(52,76)$ & $35(12,91)$ & $1(0,36)$ & $\pm 0,33 \%$ \\
\hline Todos os parâmetros como rotina * & $38(14,02)$ & & & & $\pm 0,17 \%$ \\
\hline
\end{tabular}

* = Os valores representam o número de respostas em cada categoria com os respectivos percentuais, entre parênteses

Tabela V - Rotinas de Monitorização na SRPA dos Parâmetros Indicados para Pacientes Selecionados

\begin{tabular}{|c|c|c|c|c|c|}
\hline Parâmetros & Rotina & Pacientes Selecionados & Nunca & Sem Resposta & IC95\% \\
\hline Eletrocardiograma (cardioscópio) * & $148(54,61)$ & $114(42,06)$ & $9(3,32)$ & $0(0)$ & $\pm 0,35 \%$ \\
\hline Temperatura * & $105(38,74)$ & $123(45,38)$ & $43(15,86)$ & $0(0)$ & $\pm 0,36 \%$ \\
\hline Força muscular com estimulador de nervo * & $7(2,58)$ & $107(39,48)$ & $156(57,56)$ & $1(0,36)$ & $\pm 0,35 \%$ \\
\hline Débito urinário/capacidade de micção * & $89(32,84)$ & $179(66,05)$ & $3(1,10)$ & $0(0)$ & $\pm 0,33 \%$ \\
\hline Drenagem e sangramento * & $130(47,97)$ & $137(50,55)$ & $4(1,47)$ & $0(0)$ & $\pm 0,36 \%$ \\
\hline
\end{tabular}

* = Os valores representam o número de respostas em cada categoria com os respectivos percentuais, entre parênteses

Tabela VI - Condutas Terapêuticas na SRPA dos Participantes

\begin{tabular}{|c|c|c|c|c|c|}
\hline & Rotina & Pacientes Selecionados & Nunca & Sem Resposta & IC95\% \\
\hline Oxigenioterapia * & $155(57,19)$ & $116(42,8)$ & $0(0)$ & $0(0)$ & $\pm 0,36 \%$ \\
\hline Antieméticos profiláticos * & $110(40,59)$ & $149(54,98)$ & $12(4,42)$ & $1(0,36)$ & $\pm 0,36 \%$ \\
\hline Tratamento de calafrios pós-anestésicos * & $61(22,50)$ & $180(66,42)$ & $29(10,70)$ & $1(0,36)$ & $\pm 0,25 \%$ \\
\hline
\end{tabular}

* = valores representam o número de respostas em cada categoria com os respectivos percentuais, entre parênteses

Tabela VII - Critérios de Alta da SRPA dos Participantes

\begin{tabular}{|c|c|c|c|c|c|}
\hline Critérios de Alta & Rotina & Pacientes Selecionados & Nunca & Sem Resposta & $\mathrm{IC} 95 \%$ \\
\hline Escore de Aldrete-Kroulik $\geq 9$ antes da alta da SRPA * & $195(71,95)$ & $46(16,97)$ & $25(9,22)$ & $5(1,84)$ & $\pm 0,29 \%$ \\
\hline $\begin{array}{l}\text { Regressão do bloqueio sensitivo a L1 em pacientes internados } \\
\text { submetidos a anestesia neuroaxial * }\end{array}$ & $142(52,93)$ & $84(30,99)$ & $34(12,54)$ & $11(4,05)$ & $\pm 0,36 \%$ \\
\hline $\begin{array}{l}\text { Capacidade de movimentação dos membros inferiores em } \\
\text { pacientes internados submetidos a anestesia neuroaxial * }\end{array}$ & $205(75,64)$ & $50(18,45)$ & $9(3,32)$ & $7(2,58)$ & $\pm 0,27 \%$ \\
\hline Micção mandatória antes da alta de pacientes ambulatoriais * & $137(50,55)$ & $82(30,25)$ & $44(16,23)$ & $8(2,95)$ & $\pm 0,36 \%$ \\
\hline $\begin{array}{l}\text { Retorno da sensibilidade perineal em pacientes ambulatoriais } \\
\text { submetidos a anestesia neuroaxial * }\end{array}$ & $131(48,33)$ & $47(17,34)$ & $81(29,88)$ & $12(4,42)$ & $\pm 0,36 \%$ \\
\hline
\end{tabular}

* = Os valores representam o número de respostas em cada categoria com os respectivos percentuais, entre parênteses

1. A disponibilidade de equipamentos de reanimação cresceu progressivamente para os anestesiologistas atuando nas regiões norte/nordeste, centro-oeste, sul e sudeste $[1,54(1,15-20,6)]$, o mesmo ocorrendo com a disponibilidade de estimulador de nervo periférico $[1,36$ $(1,09-1,71)]$ e dispositivos de aquecimento ativo $[1,37$ $(1,10-1,71)]$.
2. A existência de PRM na instituição associou-se com os seguintes itens: existência de Centro Cirúrgico Ambulatorial $[2,26(1,21-4,22)]$, existência de anestesiologista de plantão nas 24 horas [2,45 (1,20 - 5)], existência de anestesiologista plantonista exclusivo para a SRPA $[4,26(1,67-10,85)]$, disponibilidade de equipamento de reanimação na SRPA $[2,62(1,21-5,67)]$, existência de 
Tabela VIII - Cumprimento de Exigências Médico-Legais

\begin{tabular}{|c|c|c|c|c|c|}
\hline & Rotina & Pacientes selecionados & Nunca & Sem Resposta & IC95\% \\
\hline Registro no prontuário dos parâmetros aferidos na SRPA * & $229(84,50)$ & $0(0)$ & $40(14,76)$ & $2(0,73)$ & $\pm 0,91 \%$ \\
\hline Formulário próprio para registro do escore de Aldrete-Kroulik & $185(68,36)$ & $0(0)$ & $85(31,36)$ & $1(0,36)$ & $\pm 0.31 \%$ \\
\hline Tempo mínimo de permanência na SRPA * & $163(60,14)$ & $80(29,52)$ & $28(10,33)$ & $0(0)$ & $\pm 0,35 \%$ \\
\hline $\begin{array}{l}\text { Alta da SRPA delegada a enfermeiro ou técnico de } \\
\text { enfermagem* }\end{array}$ & $11(4,05)$ & $64(23,61)$ & $192(70,24)$ & $4(1,47)$ & $\pm 0,30 \%$ \\
\hline Acompanhante responsável para pacientes ambulatoriais * & $237(87,45)$ & $16(5,90)$ & $8(2,95)$ & $10(3,69)$ & $\pm 0,16 \%$ \\
\hline $\begin{array}{l}\text { Orientações pós-anestésicas escritas para pacientes } \\
\text { ambulatoriais* }\end{array}$ & $72(26,56)$ & $87(32,10)$ & $94(34,68)$ & $18(6,64)$ & $\pm 0,28 \%$ \\
\hline
\end{tabular}

* = Os valores representam o número de respostas em cada categoria com os respectivos percentuais, entre parênteses

estimulador de nervo periférico $[3,59(2,15-6,01)]$ e de dispositivo de aquecimento ativo $[4(2,14-7,49)]$. A probabilidade de monitorização rotineira da freqüência respiratória também foi maior entre anestesiologistas que atuam em instituições com PRM $[1,72(1,03-2,86)]$. A monitorização de força muscular seletivamente, por estimulador de nervo periférico, também foi mais provável entre os anestesiologistas destas instituições [3,37 $(1,96-5,80)]$.

3. Anestesiologistas que atuam em instituições universitárias, diferenciaram-se dos que atuam em instituições não universitárias pelos seguintes itens: monitorização da força muscular por exame clínico [1,72 (1,02 - 2,9)], uso seletivo de oxigenioterapia $(0,52(0,30-0,92)]$, tratamento rotineiro de calafrios $[0,40(0,19-0,85)]$, monitorização seletiva da diurese $[0,51(0,29-0,88)]$ e dos fluidos de drenagem e sangramento $[0,53(0,30-0,94)]$.

4. Anestesiologistas que atuam em instituições de caráter público diferenciam-se dos que atuam em instituições privadas pelos seguintes itens: disponibilidade de dispositivos de aquecimento ativo $[0,4(0,21-0,75)]$ e exigência de acompanhante responsável para alta de pacientes ambulatoriais $[0,41(0,18-0,90)]$.

5. Houve aumento progressivo dos percentuais de monitorização rotineira do nível de consciência, de acordo com o nível de experiência profissional $[1,85(1,23-2,79)]$, o mesmo ocorrendo com a monitorização rotineira de náuseas e vômitos [1,71 (1,18 - 2,48)]. Anestesiologistas mais experientes tenderam a utilizar menos o escore de Aldrete-Kroulik maior ou igual a 9 pontos como critério de alta do que os menos experientes [0,59 $(0,36-0,94)]$.

6 . Os anestesiologistas portadores de TSA diferenciaram-se dos não portadores apenas pela maior probabilidade de monitorizar rotineiramente a força muscular pelo exame clínico [1,92 $(1,13$ - 3,25)] e de utilizar o escore de Aldrete-Kroulik maior ou igual a 9 pontos como critério de alta da SRPA [2,62 (1,32 - 5,18)].

7. A existência de formulário próprio para o registro do escore de Aldrete-Kroulik associou-se com maior probabilidade de monitorização rotineira da dor pós-operatória [2,13 (1,21 - 3,73)], da ocorrência e intensidade de náu- seas e vômitos [2,64 (1,54 - 4,54)] e da monitorização rotineira de todos os parâmetros considerados essenciais [3,51 (1,31 - 9,39)]. A utilização do escore de AldreteKroulik maior ou igual a nove também foi maior entre anestesiologistas que têm este formulário em suas instituições $[3,41(3,22-11,36)]$. Estes também se diferenciaram dos que não têm o formulário disponível pela probabilidade de monitorização seletiva da força muscular por estimulador de nervo periférico $[2,31(1,24$ $4,29)]$ e da drenagem e sangramento $[0,36(0,20-0,63)]$.

\section{DISCUSSÃO}

A técnica de amostragem probabilística aleatória simples utilizada neste estudo é a mais indicada para pesquisas de caráter descritivo e conclusivo, porque garante a cada membro da população em estudo iguais chances de participar da amostragem ${ }^{3,4}$. Neste estudo, procurou-se garantir a representatividade calculando, a priori, o número de elementos da amostra e considerando uma taxa de retorno baseada na literatura $^{2-5}$. A escolha dos participantes por geração de números aleatórios, em processo informatizado, visou eliminar qualquer tendenciosidade no envio dos questionários. A representatividade se confirmou pelos intervalos de $95 \%$ de confiança inferiores a $1 \%$ das percentagens de respostas em cada item da pesquisa. O nível de $95 \%$ confiança significa que, se forem selecionadas 100 novas amostras aleatórias da população de anestesiologistas brasileiros e calculados 100 diferentes intervalos de $95 \%$ de confiança das percentagens de resposta, pode-se esperar que os percentuais obtidos nesta amostra estejam contidos nestes intervalos de confiança, em 95 das novas amostras pesquisadas ${ }^{4}$. Assim, os intervalos de $95 \%$ de confiança inferiores a $1 \%$ obtidos nesta pesquisa garantem a homogeneidade e a representatividade da amostra utilizada, permitindo extrapolação dos resultados para a população de anestesiologistas brasileiros, dentro da limitação de $95 \%$ de confiança, com erro máximo de $5 \%$ nos valores percentuais.

Em ordem decrescente de prioridade, os parâmetros monitorizados rotineiramente pelo participantes foram: pressão arterial, freqüência cardíaca, saturação periférica da oxihe- 
moglobina, nível de consciência, patência das vias aéreas, freqüência respiratória, náuseas e vômitos, dor pós-operatória e força muscular pelo exame clínico. Conforme mostra a figura 1, houve decréscimo progressivo da freqüência cumulativa de monitorização rotineira, à medida que o número de parâmetros aumentou.

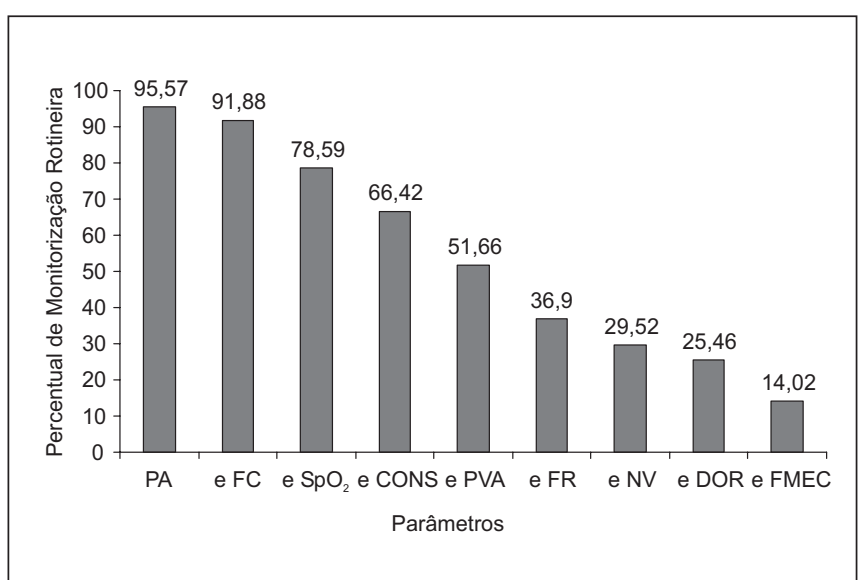

Figura 1 - Efeito da Adição Progressiva de Parâmetros de Monitorização sobre o Percentual Cumulativo de Monitorização Rotineira. Observa-se que o percentual diminui à medida em que parâmetros com menores percentuais individuais são adicionados. A letra "e" antes da sigla do parâmetro representa a adição do parâmetro aos anteriores. PA = pressão arterial, $\mathrm{FC}=$ freqüência cardíaca, $\mathrm{SpO}_{2}$ = saturação periférica da oxihemoglobina, $\mathrm{CONS}=$ nível de consciência, $\mathrm{PVA}=$ patência das vias aéreas, $F R=$ freqüência respiratória, $N V$ = náuseas e vômitos pós-operatórios, DOR = dor pós-operatória, $\mathrm{FMEC}=$ força muscular avaliada pelo exame clínico

Embora tenha havido associações significativas entre a existência de PRM na instituição e a monitorização rotineira da freqüência respiratória, entre o caráter universitário da instituição e o porte de TSAe a monitorização rotineira da força muscular pelo exame clínico, entre o nível de experiência profissional e a monitorização rotineira do nível de consciência e das náuseas e vômitos, nenhum destes fatores influenciou significativamente a freqüência de monitorização rotineira de todos os parâmetros. Por outro lado, a existência de formulário específico para o registro do escore de Aldrete-Kroulik se associou com probabilidade 3,5 vezes maior de monitorização rotineira destes parâmetros, por aumentar o registro de dor pós-operatória e da intensidade de náuseas e vômitos.

Este estudo evidenciou que, entre anestesiologistas que atuam em instituições universitárias, a opção por uso seletivo de oxigenioterapia é menor do que entre anestesiologistas que atuam em instituições não universitárias, uma vez que, entre os primeiros, o uso de oxigenioterapia é mais freqüentemente rotineiro do que entre os últimos.

A incorporação do oxímetro de pulso na monitorização pós-anestésica permite a utilização seletiva de oxigenioterapia, ou seja, oxigênio é administrado apenas para pacientes que apresentem dessaturação da oxihemoglobina. Por exemplo, na SRPAde um hospital geral, oxigênio foi adminis- trado apenas a pacientes que apresentaram $\mathrm{SpO}_{2}$ menor que $94 \%$, o que correspondeu a $37 \%$ dos pacientes estudados, resultando em substancial redução dos custos ${ }^{6}$. Em pacientes submetidas a laparoscopias ginecológicas, utilizando o mesmo critério, oxigênio foi administrado a $15 \%$ dos casos $^{7}$. Em outro estudo ${ }^{8}$, os fatores de risco para hipoxemia pós-operatória $\left(\mathrm{SpO}_{2}<92 \%\right)$ foram indicadores de hipoxemia: idade maior que 55 anos $[4,32(1,70-10,95)], \mathrm{SpO}_{2}$ pré-operatória menor que $95 \%[7,47(1,50$ - 37,11)], anestesia geral com enflurano $[14,53(2,54-82,93)]$ e hipoventilação detectada clinicamente [34,82 (11,46 - 105,84)]. Este último fator foi obtido pelo exame clínico da ventilação, incluindo a freqüência respiratória e a patência das vias aérea.

Em relação ao tratamento de calafrios pós-operatórios, anestesiologistas de hospitais universitários foram mais seletivos do que os que trabalham em instituições não universitárias. Os calafrios pós-operatórios ocupam o oitavo lugar em importância em uma relação de 33 eventos que deveriam ser rotineiramente evitados no período peri-operatório ${ }^{9}$. Além de causarem desconforto, os calafrios se associam com aumento de até $500 \%$ do consumo de oxigênio, o que, em pacientes coronariopatas, pode agravar o déficit de suprimento de oxigênio para o miocárdio. Os calafrios pós-operatórios causam aumento da pressão intracraniana e intra-ocular, além de aumentar a dor incisional ${ }^{10}$. Por estas razões, devem ser tratados rotineiramente, com a administração de meperidina, a droga considerada a mais efetiva para seu controle. Outras opções igualmente eficazes são a clonidina e o doxapram ${ }^{11}$. A administração de $30 \mathrm{mg}$ de meperidina por via venosa abole os calafrios, sem agravar a hipercarbia, mas não corrige a hipoxemia, razão pela qual a oxigenioterapia deve constar do tratamento ${ }^{12}$.

Anestesiologistas que atuam preferencialmente em instituições públicas relataram menor disponibilidade de dispositivos de aquecimento ativo dos que os que atuam em instituições privadas, enquanto os que atuam em instituições que possuem PRM relataram maior disponibilidade destes equipamentos do que os que atuam em instituições que não possuem PRM. A hipotermia pós-operatória, definida como temperatura central menor que $36^{\circ} \mathrm{C}$, associa-se com maior probabilidade de infecção da ferida operatória, de sangramento, de complicações cardíacas e com maior tempo de permanência na SRPA. Os fatores de risco são as faixas etárias neonatal e geriátrica, a baixa temperatura da sala cirúrgica, as queimaduras extensas, a anestesia geral combinada com bloqueio neuroaxial, a hipotermia prévia à indução anestésica, o tipo físico astênico e as grandes perdas sangüíneas ${ }^{13}$. Por isto, dispositivos de aquecimento ativo devem estar disponíveis na SRPA, para uso em pacientes selecionados. As condutas dos participantes em relação ao uso de antieméticos profiláticos, micção e ingestão hídrica necessárias antes da alta de pacientes ambulatoriais não foram influenciadas por nenhuma das variáveis independentes utilizadas neste estudo, refletindo homogeneidade de condutas. As náuseas e vômitos pós-operatórios estão entre os fatores que retardam a alta de pacientes ambulatoriais ${ }^{14}$ e ocupam o $2^{\circ}$ e $4^{\circ}$ lugares, respectivamente, entre problemas que deve- 
riam ser evitados no período peri-anestésico ${ }^{9}$. O risco de náuseas e vômitos diminui com o avanço da idade, no sexo masculino e entre pacientes fumantes. A história prévia de náuseas e vômitos pós-operatórios, as cirurgias de longa duração e as acompanhadas de dor pós-operatória intensa se associam com maiores riscos de ocorrência de náuseas e vômitos pós-operatórios em pacientes ambulatoriais ${ }^{15}$. $\mathrm{O}$ uso per-operatório de opióides se associa com maior probabilidade de náuseas e vômitos pós-operatórios, enquanto a anestesia venosa com propofol se associa com menor probabilidade de ocorrência desta complicação ${ }^{16}$. O conhecimento destes fatores de risco permite o uso seletivo de anti-semíticos em regime profilático, contribuindo para a redução dos custos da permanência na SRPA e para a maior satisfação dos pacientes.

Em dois estudos ${ }^{17,18}$, foram identificados os seguintes fatores de risco para a ocorrência de retenção urinária pós-operatória: cirurgias anais, herniorrafias inguinais, história prévia de retenção urinária e anestesias neuroaxiais. Em ambos os estudos, a ocorrência de retenção urinária pós-operatória em pacientes sem estes fatores de risco foi de $0,5 \%$ e $0,8 \%$ e entre os pacientes com fatores de risco, variou entre $5 \%$ e $17 \%$. Assim, a baixa incidência da complicação em pacientes sem fatores de risco não justifica a exigência de que permaneçam no hospital até que urinem espontaneamente.

A exigência de que pacientes ambulatoriais sejam capazes de tolerar líquidos por via oral baseia-se na suposição de que, se tolerarem líquidos ou alimentos, serão também capazes de tolerar a medicação pós-operatória, por via oral, sem náuseas ou vômitos. Um estudo que avaliou a prevalência de náuseas e vômitos pós-operatórios tardios em pacientes que aguardaram no hospital até serem capazes de aceitar uma ingestão hídrica obrigatória e que não ingeriram líquidos antes da alta concluiu que não houve diferença entre os grupos quanto à incidência de náuseas e vômitos pós-operatórios, e que os pacientes que somente receberam alta após conseguirem ingerir líquidos demoraram mais para deambular e urinar espontaneamente, além de terem permanecido mais tempo no hospital ${ }^{19}$. Portanto, a administração de líquidos por via oral deve ser feita sob requisição do paciente, ao invés de ser uma exigência para a alta de pacientes ambulatoriais.

Os participantes também foram homogêneos quanto à utilização do critérios de alta para pacientes internados e ambulatoriais submetidos à anestesia neuroaxial. Dois aspectos merecem atenção em relação à regressão da anestesia neuroaxial. O primeiro é a possibilidade de hipotensão postural, causada pelo bloqueio do sistema nervoso simpático associado ou não à hipovolemia, e o segundo, que diz respeito somente a pacientes ambulatoriais, refere-se às funções motora e esfinctérica e à sensibilidade proprioceptiva dos membros inferiores.

Embora existam evidências de que o bloqueio simpático se dissipe mais precocemente que o bloqueio motor ${ }^{20}$, é prudente que se utilize a regressão do nível superior do bloqueio sensitivo a, pelo menos $L_{1}$, a fim de minimizar a chance de bloqueio simpático extenso, e que se faça um teste de hipo- tensão ortostática, elevando o tronco entre $45^{\circ}$ e $60^{\circ}$ e medindo a variação da pressão arterial sistólica. Reduções superiores a $20 \%$ indicam bloqueio simpático significativo ou hipovolemia e contra-indicam a alta da SRPA ${ }^{21}$. No caso de pacientes ambulatoriais, além destes, devem ser utilizados critérios que garantam o retorno da função motora, da sensibilidade proprioceptiva e da atividade esfinctérica. Por isto, é recomendado que se utilizem, como critérios de alta, o retorno da sensibilidade perineal, a capacidade de flexão plantar do pé a níveis pré-operatórios e o retorno da sensibilidade proprioceptiva do hálux como critérios para alta ${ }^{22}$.

Os pacientes que apresentam eventos cardiovasculares perioperatórios (isquemia miocárdica, arritmias, edema agudo de pulmão) e os portadores de insuficiência cardíaca tendem a permanecer mais tempo no hospital após cirurgias ambulatoriais ${ }^{12}$. Nestes pacientes, o uso do cardioscópio pode auxiliar na identificação precoce e tratamento de algumas destas complicações.

Este estudo identificou uma baixa percentagem de existência de monitores de função neuromuscular no locais de trabalho dos participantes, influenciada positivamente pela existência de PRM na instituição e pela existência de formulário para registro do escore de Aldrete-Kroulik. A prevalência de bloqueio neuromuscular residual depende do critério utilizado para diagnóstico e do bloqueador neuromuscular utilizado. Em um estudo que utilizou a relação $T_{4} / T_{1}$ da seqüência de quatro estímulos inferior a 0,8 como definição de bloqueio neuromuscular residual, $64 \%, 52 \%$ e $39 \%$ dos pacientes que receberam vecurônio, atracúrio ou rocurônio, respectivamente, apresentaram bloqueio neuromuscular residual, detectável também pela incapacidade de manter a elevação da cabeça por cinco segundos ${ }^{23}$. Em outro estudo, a prevalência de bloqueio neuromuscular residual, definida como relação T4/T1 inferior a 0,7 , foi significativamente maior entre os pacientes que receberam pancurônio (26\%) do que entre os que receberam vecurônio ou atracúrio $(5,3 \%)$. Além disto, entre os pacientes que receberam pancurônio e apresentaram bloqueio neuromuscular residual, $16,9 \%$ apresentaram complicações pulmonares pós-operatórias ${ }^{24}$. A presença de diplopia ou disfagia, a incapacidade de cerrar fortemente os dentes ou de manter aperto de mão ou elevação da cabeça por, pelo menos, 5 segundos indicam a presença de bloqueio neuromuscular residual ${ }^{25}$. Nessas situações, os monitores quantitativos de função neuromuscular por acelerometria, mecanografia ou eletromiografia podem quantificar a profundidade do bloqueio neuromuscular e monitorizar sua reversão.

Aproximadamente $15 \%$ dos participantes não registram os dados clínicos pós-operatórios no prontuário. É recomendável que a documentação de todas as fases do procedimento anestésico seja feita meticulosamente, pois este registro se constitui em valiosa peça de defesa, em caso de ação judicial. A delegação da alta da SRPA a enfermeiros ou técnicos de enfermagem, que é rotina para $4,05 \%$ dos anestesiologistas e é permitida para pacientes selecionados, por $23,61 \%$, está em desacordo com a normatização vigente, segundo a qual, os critérios para a alta da SRPA são responsabilidade do 
anestesiologista responsável ou do anestesiologista plantonista a quem tenha sido delegado o cuidado pós-anestésico. Em relação à necessidade de acompanhante maior e responsável para alta de pacientes ambulatoriais, $87,45 \%$ dos anestesiologistas age de acordo com a legislação vigente. Entretanto, anestesiologistas que atuam em instituições públicas cumprem esta determinação menos freqüentemente do que os que atuam em instituições privadas. Somente $26,56 \%$ dos participantes fornece orientações pós-anestésicas escritas a pacientes ambulatoriais. As orientações escritas e assinadas pelo médico anestesiologista, pelo paciente e/ou seu responsável em, pelo menos duas vias, uma das quais permanece no prontuário, são a prova de que o paciente e seu responsável foram advertidos das limitações físicas e psicomotoras pós-anestésicas, das possíveis complicações e da forma de manuseá-las, incluindo endereços e telefones para contato, afastando, desta forma, a possibilidade de ser o médico acusado de negligência.

Este estudo sugere que os anestesiologistas brasileiros preocupam-se com a segurança dos pacientes no período pós-anestésico imediato, conforme atestam a elevada disponibilidade de Salas de Recuperação Pós-Anestésica bem equipadas e os percentuais expressivos de monitorização de parâmetros isolados. Algumas rotinas de monitorização, especialmente da dor e das náuseas e vômitos pós-operatórios, precisam ser implementadas, de acordo com as novas diretrizes de cuidados pós-anestésicos e os novos critérios de alta para pacientes ambulatoriais ${ }^{26-29}$. A utilização de formulários específicos para o registro dos parâmetros pós-anestésicos pode ser uma forma eficaz de implementar a monitorização de um maior número de parâmetros pós-anestésicos. Deve ser dada maior atenção ao fornecimento de orientações escritas para pacientes ambulatoriais e seus responsáveis.

\section{AGRADECIMENTOS}

Aos anestesiologistas e médicos residentes de todo o território nacional pelas valiosas contribuições.

\section{Postanesthetic Routines of Brazilian Anesthesiologists}

Getúlio Rodrigues de Oliveira Filho, TSA, M.D.

\section{INTRODUCTION}

Postanesthetic care encompasses monitoring and treatment activities to manage patients after an anesthetic-surgical procedure, during different anesthetic recovery steps. Most times, initial anesthetic recovery (step 1 ) is still in the operating room when the primary objective is to reestablish respiratory activity and reflexes, cardiovascular stability and muscle strength. In a second step (step II) in the Post-Anesthetic Recovery Unit (PACU), the same parameters are observed under clinical and instrumental monitoring, in addition to consciousness level. Patients submitted to neuraxial blocks remain in the PACU until sympathetic block recovery. It is during this step that postoperative pain, nausea and vomiting, shivering, urinary retention and abnormal bleedings play a fundamental role because their treatment implies more comfort and safety to patients. Patients submitted to outpatient procedures go through a third anesthetic recovery step (step III) still in the PACU or in an adequate outpatient surgery environment, where ambulation, spontaneous micturition, tolerance to oral fluids, pain, nausea and vomiting control with oral medication are assured ${ }^{1}$.

The American Society of Anesthesiologists (ASA) has recently published a report on a workgroup put together to develop practical guidelines for postoperative care ${ }^{2}$, the recommendations of which are summarized below:

1. PACU should have equipment for cardiopulmonary resuscitation;

2. There shall be an anesthesiologist available to treat PACU intercurrences;

3. The following parameters should be routinely monitored in the PACU: respiratory rate, airway patency, peripheral oxygen hemoglobin saturation $\left(\mathrm{SpO}_{2}\right)$, heart rate, blood pressure, neuromuscular function by clinical evaluation, mental status, pain and postoperative nausea and vomiting;

4. In patients selected by risk factors or clinical status, ECG by cardioscopy, neuromuscular function by peripheral nerve stimulator, temperature, urinary output, micturition ability, drains or tubes output and/or postoperative bleeding should also be monitored;

5. Anti-emetics should be used to prevent nausea and vomiting in patients selected by risk factors and should be routinely used for their treatment;

6. Oxygen therapy should be used in patients selected by risk factors for hypoxemia or its treatment;

7. Active heating devices (forced air) should be used to prevent and treat hypothermia;

8. Postoperative shivering should be routinely treated. Meperidine is the drug of choice;

9. Requirement for patients to urinate or ingest fluids before leaving the hospital should be only applied to patients with risk factors for urinary retention or nausea and vomiting, respectively;

10. Post-anesthetic observation period should be customized according to the magnitude of the risk for cardiopulmonary depression, so a minimum PACU stay period is not justified;

11. Ambulatory patients should only leave the hospital escorted by an adult in charge and after receiving, both patients and escorts, written instructions on postanesthetic care and ways to contact the hospital in case of complications. 
In Brazil, the requirement of PACUs in hospitals was determined by the Administrative Rule 400 of the Ministry of Health, in 1977. Resolution CMF 1363/93, dealing with safety in anesthesia, establishes in its article VI that "all patients should be transferred to the post-anesthetic recovery unit after surgery", and in article VIII that "discharge criteria during the post-anesthetic recovery period are a non transferable responsibility of the anesthesiologist". Resolution CFM 1409/94, dealing with outpatient surgery normalization, provides in its article III that conditions for outpatient discharge are: time and space orientation, vital signs stability for at least 60 minutes, lack of nausea or vomiting, lack of respiratory difficulties, ability to ingest fluids, locomotion ability similar to preoperative ability, minimum or non existent operative bleeding, lack of severe pain and urinary retention. It also provides that patient and escort of age and responsible should receive orally and in writing, instructions about post-anesthetic and postoperative care, as well as where to call in case of intercurrences.

This study aimed primarily at determining:

1. PACU equipment availability in institutions where participants have their primary activities;

2. How participants monitor parameters considered routine and those indicated for selected patients;

3. In which circumstances oxygen therapy, nausea and vomiting prevention and postoperative shivering control are used;

4. How discharge criteria proposed by the literature and current legislation for admitted or ambulatory patients submitted to general or neuraxial anesthesias are used;

5. How medical-legal requirements related to post-anesthetic recovery are followed.

Secondarily, the study aimed at studying the effects of geography, institution characteristics (legal regimen, university hospital and the existence of a Medical Residence in Anesthesiology Program (MRP), existence of forms to record Aldrete-Kroulik score and professional qualification (professional experience and Superior Title in Anesthesiology (TSA)) on human and material resources availability, PACU monitoring and treatment routines and compliance with medical-legal requirements.

\section{METHODS}

Through query on SBA portal (www.sba.com.br), 2246 electronic addresses of members of the Brazilian Society of Anesthesiology (SBA) were obtained via Internet. Sample size it was estimated based on a previous study ${ }^{2}$ where at least $80 \%$ of participants would consider routine the monitoring of heart rate, airway patency, $\mathrm{SpO}_{2}$, respiratory rate, blood pressure, muscle strength, consciousness level, postoperative pain intensity and nausea and vomiting. For an acceptable error of $5 \%$ and $95 \%$ confidence intervals, sample size was equal to 256 . Considering an estimated return rate of $25 \%{ }^{2-4}$,
1024 was considered the minimum number of queried anesthesiologists.

By simple randomized probabilistic sampling, 1123 anesthesiologists were chosen with the likelihood of $1: 2$ for the addresses to be picked from the list ${ }^{3-4}$.

The e-mailed message had an introduction describing the objectives of the survey, followed by the questionnaire. Questions were presented in four blocks.

Block I contemplated the following items:

1. Region - North, Northeast, Midwest, Southeast or South;

2. Professional experience - resident physician, anesthesiologist for less than 5 years or anesthesiologist for 5 years or more;

3. Superior Title in Anesthesiology - yes or no;

4. Institution's primary profile - public, university or not; or private, university or not;

5. Existence of a Medical Residence in Anesthesiology Program in the Institution - yes or no;

6. PACU location in the Operating Center, existence of an operating center exclusively for outpatient procedures, recovery room exclusively for outpatient procedures, anesthesiologist on duty 24 hours in the institution, anesthesiologist on duty 24 hours exclusively in the PACU, resuscitation equipment in the PACU including defibrillator - yes or no;

7. Number of rooms in the Operating Center, PACU, Outpatient Procedure Unit and Recovery Room of the Outpatient Procedure Unit.

Block II requested participants to check the situation (routine, selected patients or never) in which each of the following parameters is monitored in the PACU: respiratory rate, airway patency, peripheral oxygen saturation $\left(\mathrm{SpO}_{2}\right)$, heart rate, blood pressure, ECG tracing with cardioscope, muscle strength by physical evaluation, muscle strength by peripheral nerve stimulator, mental status or consciousness level, temperature, postoperative pain intensity, frequency and intensity of nausea and vomiting, urinary output, drainage and bleeding. This block has also asked whether parameters are recorded and if there is an adequate form available in their institutions to record Aldrete-Kroulik scores.

Block III requested participants to classify the use and availability of cardioscope, pulse oximeter, neuromuscular function monitor, aspirator, oxygen source and active heating equipment to be used in the PACU, according to the following categories: used/routinely available, used/available for selected patients, or non used/unavailable.

Block IV requested participants to check one alternative (used as routine, used in selected patients or never used) that would reflect their position faced to the following PACU approaches:

1. Oxygen therapy;

2. Prophylactic anti-emetics;

3. Pharmacological treatment of postanesthetic shivering with meperidine and oxygen; 
4. Mandatory micturition before discharging ambulatory patients;

5. Escorts responsible for ambulatory patients;

6. Minimum pre-established PACU stay;

7. Aldrete-Kroulik score equal to or above 9 before PACU discharge;

8. Regression of upper sensory block to at least L1 before discharging patients submitted to neuraxial blocks;

9. Ability to move lower limbs before discharging patients submitted to neuraxial blocks;

10. Written postanesthetic recommendations to ambulatory patients;

11. PACU discharge delegated to nurse or trained nursing technician.

The questionnaire ended with a statement of consent that supplied data could be published in a scientific paper, not disclosing the origin which should be informed, in addition to instructions on how to answer the message.

Statistical analysis had two stages. The first, aiming at meeting primary objectives, was a simple tabulation of answers and computation of respective percentages. The next stage was exploratory data analysis aiming at meeting secondary objectives. Each item of the questionnaire (dependent variable) was categorized according to the following independent variables: a. geographic region (North/Northeast, Midwest, South and Southeast);

b. participants' professional qualification: level of professional experience (Physician in Specialization, Anesthesiologist for less than 5 years, anesthesiologist for 5 years or more) and Superior Title in Anesthesiologist (holder or not).

c. participant institution's profile: university or not, public or private, existence or not of MRP, existence or not of a form to record Aldrete-Kroulick scores.

Categoric variables were submitted to Chi-square tests and ordinal variables were submitted to Mann-Whitey or Kruskal-Wallis tests, followed by retrograde logistic regression. Level of significance was $5 \%$.

\section{RESULTS}

From 1123 questionnaires, 104 were returned for unknown address. From 1019 mailed questionnaires, 294 were answered, corresponding to $28.85 \%$ response rate. From those answering the questionnaire, 23 have not agreed with the use of data for publishing purposes so, the analysis of results was performed with 271 participants $(26.59 \%$ of received questionnaires).

Simple tabulation of responses is shown in tables I to VIII.

Table I - General Sample Profile

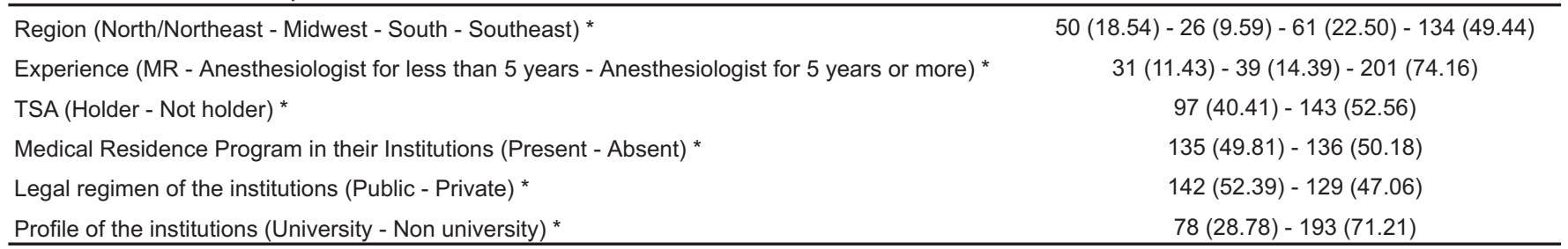

${ }^{*}=$ Values represent the number of answers in each category with respective percentages, in brackets

Table II - Profile of Institutions where Participants Work

\begin{tabular}{|c|c|c|c|}
\hline Profile & Yes & No & No Reply \\
\hline PACU within the Operating Center * & $254(93.72)$ & $16(5.9)$ & $1(0.36)$ \\
\hline Operating Center exclusively for outpatient procedures * & $95(35.05)$ & $176(64.94)$ & $0(0)$ \\
\hline Recovery Room exclusively for outpatient procedures * & $74(29.30)$ & $197(72.69)$ & $0(0)$ \\
\hline Anesthesiologist on duty 24 hours * & $206(76.01)$ & $63(23.24)$ & $2(0.73)$ \\
\hline Anesthesiologist exclusively on duty for PACU * & $40(17.76)$ & $228(84.13)$ & $3(1.10)$ \\
\hline Number of Operating Rooms & 7 & $0-33$ & $5-10$ \\
\hline Number of PACU beds & 8 & $0-40$ & $5-10$ \\
\hline PACU beds/Operating Rooms ratio & 1 & $0-3.07$ & $0.61-1.25$ \\
\hline Number of Outpatients Operating Center rooms (OOC) & 3 & $1-16$ & $2-4$ \\
\hline Number of Outpatient Recovery Room beds (ORR) & 4 & $1-25$ & $3-6$ \\
\hline OOR beds/OOC rooms ratio & 0.5 & $0.2-5$ & 12.5 \\
\hline
\end{tabular}

* Values represent the number of answers in each category with respective percentages in brackets 
Table III - Availability of PACU Equipment in the Institutions where Participants Work

\begin{tabular}{|c|c|c|c|}
\hline & Available & Unavailable & Cl95\% \\
\hline Resuscitation equipment, including defibrillator * & $235(86.71)$ & $36(13.28)$ & $\pm 0.17 \%$ \\
\hline Oxygen source * & $267(98.52)$ & $4(1.47)$ & $\pm 0.02 \%$ \\
\hline Cardioscope * & $264(97.41)$ & $7(2.58)$ & $\pm 0.03 \%$ \\
\hline Pulse Oximetry * & $268(98.89)$ & $3(1.10)$ & $\pm 0.01 \%$ \\
\hline Peripheral nerve stimulator * & $124(45.75)$ & $147(54.24)$ & $\pm 0.36 \%$ \\
\hline Active heating device * & $162(59.77)$ & $109(40.21)$ & $\pm 0.35 \%$ \\
\hline
\end{tabular}

* $=$ Values represent the number of answers in each category with respective percentages in brackets

Table IV - PACU Monitoring Routines for Essential Parameters

\begin{tabular}{|c|c|c|c|c|c|}
\hline Parameters & Routine & Selected Patients & Never & No Reply & $\mathrm{Cl} 195 \%$ \\
\hline Blood pressure * & $259(95.57)$ & $11(4.05)$ & $1(0.36)$ & $0(0)$ & $\pm 0.06 \%$ \\
\hline Heart rate * & $257(94.83)$ & $10(3.69)$ & $0(0)$ & $4(1.47)$ & $\pm 0.07 \%$ \\
\hline $\mathrm{SpO}_{2}$ * & $226(83.39)$ & $43(15.86)$ & $0(0)$ & $2(0.73)$ & $\pm 0.20 \%$ \\
\hline Consciousness level/mental status * & $225(83.02)$ & $36(13.28)$ & $9(3.32)$ & $1(0.36)$ & $\pm 0.20 \%$ \\
\hline Airway patency * & $193(71.21)$ & $58(21.40)$ & $15(5.53)$ & $5(1.84)$ & $\pm 0.30 \%$ \\
\hline Respiratory rate * & $176(64.94)$ & $79(29.15)$ & $15(5.53)$ & $1(0.36)$ & $\pm 0.33 \%$ \\
\hline Nausea and vomiting intensity * & $174(64.20)$ & $72(26.56)$ & $22(8.11)$ & $3(1.10)$ & $\pm 0.33 \%$ \\
\hline Postoperative pain intensity * & $172(63.46)$ & $69(25.46)$ & $26(9.59)$ & $4(1.47)$ & $\pm 0.34 \%$ \\
\hline Muscle strength (clinical evaluation) * & $92(33.94)$ & $143(52.76)$ & $35(12.91)$ & $1(0.36)$ & $\pm 0.33 \%$ \\
\hline All parameters as routine * & $38(14.02)$ & & & & $\pm 0.17 \%$ \\
\hline
\end{tabular}

* = Values represent the number of answers in each category with respective percentages in brackets

Table V - PACU Monitoring Routines for Selected Patients' Parameters

\begin{tabular}{|c|c|c|c|c|c|}
\hline Parameters & Routine & Selected Patients & Never & No Reply & $\mathrm{Cl} 95 \%$ \\
\hline Electrocardiogram (cardioscope) * & $148(54.61)$ & $114(42.06)$ & $9(3.32)$ & $0(0)$ & $\pm 0.35 \%$ \\
\hline Temperature * & $105(38.74)$ & $123(45.38)$ & $43(15.86)$ & $0(0)$ & $\pm 0.36 \%$ \\
\hline Urinary outuput/micturition ability * & $89(32.84)$ & $179(66.05)$ & $3(1.10)$ & $0(0)$ & $\pm 0.33 \%$ \\
\hline Drainage and bleeding * & $130(47.97)$ & $137(50.55)$ & $4(1.47)$ & $0(0)$ & $\pm 0.36 \%$ \\
\hline
\end{tabular}

* $=$ Values represent the number of answers in each category with respective percentages in brackets

Table VI - Participants' Therapeutic Approaches in the PACU

\begin{tabular}{|c|c|c|c|c|c|}
\hline & Routine & Selected Patients & Never & No Reply & $\mathrm{Cl} 95 \%$ \\
\hline Oxygen therapy * & $155(57.19)$ & $116(42.8)$ & $0(0)$ & $0(0)$ & $\pm 0.36 \%$ \\
\hline Prophylactic anti-emetics * & $110(40.59)$ & $149(54.98)$ & $12(4.42)$ & $1(0.36)$ & $\pm 0.36 \%$ \\
\hline
\end{tabular}

* $=$ Values represent the number of answers in each category with respective percentages in brackets

Table VII - Participants' PACU Discharge Criteria

\begin{tabular}{|c|c|c|c|c|c|}
\hline Discharge Criteria & Routine & Selected Patients & Never & No Reply & $\mathrm{Cl} 95 \%$ \\
\hline Aldrete-Kroulik score $\geq 9$ before PACU discharge * & $195(71.95)$ & $46(16.97)$ & $25(9.22)$ & $5(1.84)$ & $\pm 0.29 \%$ \\
\hline $\begin{array}{l}\text { Sensory block regression to L1 in admitted patients submitted to neuraxial } \\
\text { anesthesia * }\end{array}$ & $142(52.93)$ & $84(30.99)$ & $34(12.54)$ & $11(4.05)$ & $\pm 0.36 \%$ \\
\hline $\begin{array}{l}\text { Ability to move lower limbs in admitted patients submitted to neuraxial } \\
\text { anesthesia * }\end{array}$ & $205(75.64)$ & $50(18.45)$ & $9(3.32)$ & $7(2.58)$ & $\pm 0.27 \%$ \\
\hline Mandatory micturition before discharging ambulatory patients * & $137(50.55)$ & $82(30.25)$ & $44(16.23)$ & $8(2.95)$ & $\pm 0.36 \%$ \\
\hline $\begin{array}{l}\text { Perineal sensitivity return in ambulatory patients submitted to neuraxial } \\
\text { anesthesia * }\end{array}$ & $131(48.33)$ & $47(17.34)$ & $81(29.88)$ & $12(4.42)$ & $\pm 0.36 \%$ \\
\hline
\end{tabular}

*Values represent the number of answers in each category with respective percentages in brackets 
Table VIII - Compliance with Medical-Legal Requirements

\begin{tabular}{|c|c|c|c|c|c|}
\hline & Routine & Selected Patients & Never & No Reply & IC95\% \\
\hline Record of PACU-measured parameters * & $229(84.50)$ & $0(0)$ & $40(14.76)$ & $2(0.73)$ & $\pm 0.91 \%$ \\
\hline Adequate form to record Aldrete-Kroulik scores & $185(68.36)$ & $0(0)$ & $85(31.36)$ & $1(0.36)$ & $\pm 0.31 \%$ \\
\hline Minimum PACU stay * & $163(60.14)$ & $80(29.52)$ & $28(10.33)$ & $0(0)$ & $\pm 0.35 \%$ \\
\hline PACU discharge delegated to nurse or nursing technician * & $11(4.05)$ & $64(23.61)$ & $192(70.24)$ & $4(1.47)$ & $\pm 0.30 \%$ \\
\hline Tutors in charge of ambulatory patients * & $237(87.45)$ & $16(5.90)$ & $8(2.95)$ & $10(3.69)$ & $\pm 0.16 \%$ \\
\hline Written post-anesthetic recommendations for ambulatory patients * & $72(26.56)$ & $87(32.10)$ & $94(34.68)$ & $18(6.64)$ & $\pm 0.28 \%$ \\
\hline
\end{tabular}

${ }^{*}=$ Values represent the number of answers in each category with respective percentages in brackets

Multivariate analysis has identified independent variables associated to more (odds ratio higher than 1) or less (odds ratio lower than 1) likelihood of human and material resources availability, of monitoring practices and of medical-legal requirements compliance. Significant associations are described as follows. Values in crotchets refer to odds ratio and respective $95 \%$ confidence intervals are shown in brackets:

1. Resuscitation equipment availability has progressively increased for anesthesiologists working in Northern/ Northeastern, Midwestern, Southern and Southeastern regions [1.54 (1.15 - 20.6)], the same being true for the availability of peripheral nerve stimulator [1.36 (1.091.71)] and active heating equipment [1.37 (1.10 - 1.71)].

2. The existence of Medical Residence Programs in the institution was correlated to the following items: existence of Outpatient Procedure Centers [2.26 (1.21 - 4.22)], existence of anesthesiologist on duty for 24 hours [2.45 (1.20 - 5)], existence of anesthesiologist on duty exclusively in the PACU [4.26 (1.67 - 10.85)], availability of resuscitation equipment in the PACU [2.62 (1.21 - 5.67)], existence of peripheral nerve stimulator [3.59 (2.15$6.01)$ ] and of active heating device [4 (2.14 - 7.49)]. The likelihood of routine monitoring and respiratory frequency was also higher among anesthesiologists working in institutions with MRP [1.72 (1.03 - 2.86)]. Selective muscle strength evaluation with peripheral nerve stimulator was also more likely among anesthesiologists of those institutions [3.37 (1.96 - 5.80)].

3. Anesthesiologists working in university institutions have differed from those working in non-university institutions in the following items: muscle strength monitoring by physical evaluation [1.72 (1.02 - 2.9)], selective use of oxygen therapy $[0.52(0.30-0.92)]$, routine treatment of shivering $[0.40(0.19-0.85)]$, selective monitoring of diuresis $[0.51(0.29-0.88)]$ and of drainage and bleeding [0.53 (0.30-0.94)].

4. Anesthesiologists working in public institutions have differed from those working in private institutions in the following items: availability of active heating equipment [0.4 (0.21 - 0.75)] and requirement of an escort for discharging ambulatory patients [0.41 (0.18-0.90)].
5. There was a progressive increase in routine consciousness level monitoring as a function of the level of professional experience [1.85 (1.23 - 2.79)], the same being true for routine monitoring of nausea and vomiting [1.71 (1.18 - 2.48)]. More experienced anesthesiologists tended to use less Aldrete-Kroulik score equal to or higher than 9 as discharge criteria, as compared to less experienced professionals [0.59 $(0.36-0.94)]$.

6. Superior Title in Anesthesiology professionals have differed from non-TSAs in the higher likelihood for routine monitoring of muscle strength by physical evaluation [1.92 (1.13 - 3.25)] and for using Aldrete-Kroulik score equal to or higher than 9 as PACU discharge criteria [2.62 (1.32 - 5.18)].

7. The existence of a form to record Aldrete-Kroulik scores was associated to higher likelihood of routine monitoring of postoperative pain [2.13 (1.21 - 3.73)], of the incidence and intensity of nausea and vomiting [2.64 (1.54 4.54)] and of all essential parameters [3.51 (1.31 9.39)]. The use of Aldrete-Kroulik score equal to or higher than 9 was also higher among anesthesiologists having such forms in their institutions [3.41 (3.22 - 11.36)]. They have also differed from those not having the form in the likelihood of selective monitoring of muscle strength with peripheral nerve stimulator [2.31 (1.24 - 4.29)] and of draining and bleeding [0.36 (0.20-0.63)].

\section{DISCUSSION}

Simple randomized probabilistic sampling techniques used in this study are the most adequate for descriptive and conclusive surveys because they assure to each sample member equal chances of participating in the sample ${ }^{3,4}$. This study attempted to assure representation by calculating a priori, the number of sample elements and considering a literature-based return rate ${ }^{2-5}$. The choice of participants by generating computer-aided random numbers aimed at eliminating biases in the mailing of questionnaires. Representation was confirmed by $95 \%$ confidence intervals lower than $1 \%$ of answers ratio in each surveyed item. $95 \%$ confidence interval means that if 100 new randomized samples are selected within the population of Brazilian anesthesiologists and if 100 different $95 \%$ confidence intervals are calculated 
in answers ratio, it is to be expected that percentages obtained in this sample would be within these confidence intervals in 95 out of 100 new surveyed samples ${ }^{4}$. So, $95 \%$ confidence interval below $1 \%$ obtained in this survey assure homogeneity and representation of the sample, allowing the results to be extrapolated to the population of Brazilian anesthesiologists, with the limitation of $95 \%$ confidence, and maximum error of $5 \%$ in percentage values.

In decreasing order of priority, parameters routinely monitored by participants were: blood pressure, heart rate, peripheral oxygen saturation, consciousness level, airway patency, respiratory rate, nausea and vomiting, postoperative pain and muscle strength by physical evaluation. There has been a progressive decrease in cumulative frequency of routine monitoring as the number of parameters increased, as shown in figure 1.

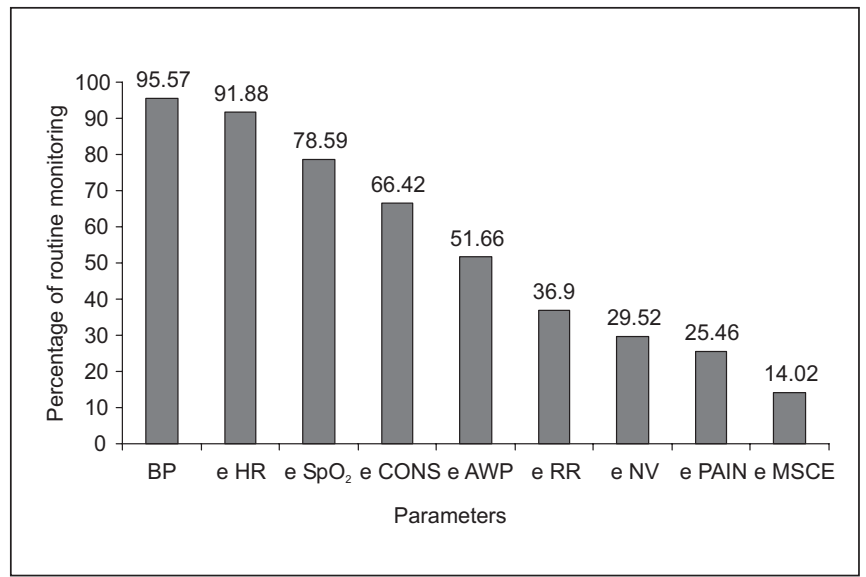

Figure 1 - Effect of progressive Addition of Monitoring Parameters on Cumulative Percentage of Routine Monitoring. It is observed that the percentage decreases as parameters with lower individual percentages are added. Letter "e" before parameter acronym represents addition of the parameter to previous parameters. $\mathrm{BP}=$ blood pressure, $\mathrm{HR}=$ Heart Rate, $\mathrm{SpO}_{2}=$ peripheral oxygen saturation, CONS = consciousness level, AWP $=$ Airway patency, $\mathrm{RR}=$ respiratory rate, NV = postoperative nausea and vomiting, PAIN = postoperative pain, MSCE = muscle strength by clinical evaluation

Although significant associations between the existence of MRP and routine respiratory rate monitoring, between the university character of the institution and TSA holders, and routine muscle strength monitoring by physical evaluation, between the level of professional experience and routine consciousness level, nausea and vomiting monitoring, none of those factors have significantly influenced the frequency of routine monitoring of all parameters. On the other hand, the existence of a specific form to record Aldrete-Kroulik scores was associated to a 3.5 times higher likelihood of routine monitoring of such parameters, for increasing the recording of postoperative pain and nausea and vomiting intensity.

This study has shown that among anesthesiologists working in university institutions, the selective use of oxygen therapy is lower than among anesthesiologists working in non-university institutions, since among the former, oxygen therapy is more often routine than among the latter.

The incorporation of pulse oximetry in postanesthetic monitoring allows the selective use of oxygen therapy, that is, oxygen is administered only to patients with oxyhemoglobin desaturation. For example, in a general hospital PACU, oxygen was administered only to patients with $\mathrm{SpO}_{2}$ below $94 \%$, corresponding to $37 \%$ of patients, resulting in significant cost reduction ${ }^{6}$. In patients submitted to gynecological laparoscopies and using the same criteria, oxygen was administered to $15 \%$ of cases ${ }^{7}$. In a different study ${ }^{8}$ on risk factors for postoperative hypoxemia $\left(\mathrm{SpO}_{2}<92 \%\right)$, indicators were: age above 55 years [4.32 (1.70 - 10.95)], preoperative $\mathrm{SpO}_{2}$ below $95 \%$ [7.47 (1.50 - 37.11)], general anesthesia with enflurane [14.53 (2.54 - 82.93)] and clinically detected hypoventilation [34.82 (11.46 - 105.84)]. This latter factor was obtained by clinical ventilation evaluation, including respiratory rate and airway patency.

As to postoperative shivering control, university hospital anesthesiologists were more selective as compared to those working in non-university institutions. Postoperative shivering is the $8^{\text {th }}$ out of 33 important events which should be routinely avoided in the perioperative period ${ }^{9}$. In addition to bringing discomfort, shivering is associated to up to $500 \%$ increase in oxygen consumption which, in cardiac patients, may worsen the deficit of oxygen supply to the myocardium. Postoperative shivering increases intracranial and intraocular pressures, in addition to worsening incision pain ${ }^{10}$. For this reason it should be routinely treated with meperidine, considered the most effective drug for its control. Other equally effective options are clonidine and doxapram ${ }^{11}$. Intravenous $30 \mathrm{mg}$ meperidine controls shivering without worsening hypercarbia, but does not correct hypoxemia, reason why oxygen therapy should be part of the treatment ${ }^{12}$.

Anesthesiologists primarily working in public institutions have reported lower availability of active heating devices as compared to those working in private institutions, while those working in institutions with MRP have reported higher availability of such equipment as compared to those working in institutions without MRP. Postoperative hypothermia, defined as central temperature lower than $36^{\circ} \mathrm{C}$, is associated to a higher likelihood of surgical wound infections, bleedings, cardiac complications and longer PACU stay. Risk factors are neonatal and geriatric age brackets, low operating room temperature, extensive burns, general anesthesia combined with neuraxial block, pre anesthetic hypothermia, asthenia and major blood losses ${ }^{13}$. For those reasons, active heating devices should be available in the PACU to be used in selected patients.

Participants' approach for prophylactic anti-emetics, and micturition and fluid ingestion before discharging ambulatory patients was not influenced by any independent variable used in this study, reflecting homogeneity of approaches. Postoperative nausea and vomiting are among factors delaying ambulatory patients discharge ${ }^{14}$ and are the $2^{\text {nd }}$ and $4^{\text {th }}$ problem, respectively, to be avoided in the peri-anesthetic 
period $^{9}$. The risk for nausea and vomiting decreases with increased age, in male patients and among smokers. Previous history of postoperative nausea and vomiting, long surgeries and those followed by severe postoperative pain are associated to an increased risk for postoperative nausea and vomiting in ambulatory patients ${ }^{15}$. Perioperative opioids are associated to a higher incidence of postoperative nausea and vomiting, while intravenous propofol is associated to a lower incidence of such complication ${ }^{16}$. The understanding of those risk factors allows for the selective use of prophylactic anti-emetic drugs, contributing to decrease PACU stay costs and to higher patients' satisfaction.

Two different studies ${ }^{17,18}$ have identified the following risk factors for postoperative urinary retention: anal surgeries, inguinal herniorrhaphies, previous urinary retention history, and neuraxial anesthesias. In both studies, the incidence of postoperative urinary retention in patients without those risk factors was $0.5 \%$ and $0.8 \%$, and among risk factor patients it has varied from $5 \%$ to $17 \%$. So, the low incidence of complications among patients without risk factors does not justify the requirement of remaining in the hospital until spontaneous micturition.

The requirement that ambulatory patients should be able to tolerate oral fluids is based on the assumption that, if they tolerate fluids or food, they will also be able to tolerate postoperative oral medication, without nausea or vomiting. A study evaluating the incidence of late postoperative nausea and vomiting in patients remaining in the hospital until they were able to accept fluid ingestion and those not ingesting fluids before discharge has found no differences between groups in the incidence of postoperative nausea and vomiting, and that patients who were only discharged after being able to ingest fluids took longer to ambulate and spontaneously urinate, in addition to having remained longer in the hospital ${ }^{19}$. So, oral fluids should only be administered at patients' request and should not be a requirement for ambulatory patients discharge.

Participants were also homogeneous in discharge criteria for ambulatory and admitted patients submitted to neuraxial anesthesia. Two aspects of neuraxial anesthesia recovery should be highlighted: first is the possibility of postural hypotension caused by sympathetic system block, associated or not to hypovolemia; second, and related only to ambulatory patients, has to do with motor and sphincter functions and lower limbs proprioceptive sensitivity.

Although there are evidences that sympathetic block recovery is faster as compared to motor block ${ }^{20}$, it is prudent to use upper sensory block level regression to at least $L_{1}$, to minimize the chance for extensive sympathetic block, and that an orthostatic hypotension test is performed raising the body between $45^{\circ}$ and $60^{\circ}$ and measuring systolic pressure variation. Decreases above $20 \%$ indicate significant sympathetic block or hypovolemia, and counterindicate PACU discharge ${ }^{21}$. In addition to these evaluations, criteria should be applied to ambulatory patients to assure the return of motor function, of proprioceptive sensitivity and of sphincter activity. So, it is recommended that recovery of perineal sensitivity, ability to flexion the foot to preoperative levels and recovery of hallux proprioceptive sensitivity are used as discharge criteria ${ }^{22}$. Patients with perioperative cardiovascular events (myocardial ischemia, arrhythmias, acute lung edema) and those with heart failure tend to remain longer in the hospital after outpatient procedures ${ }^{12}$. In those patients, cardioscopy may help the early identification and treatment of some of these complications. This study has identified a low percentage of neuromuscular function monitors in participants' working places, positively influenced by MRPs and by the existence of forms to record Aldrete-Kroulik scores. The prevalence of residual neuromuscular block depends on diagnostic criteria and the neuromuscular blocker. In a study using TOF stimulation $\mathrm{T}_{4} / \mathrm{T}_{1}$ ratio below 0.8 to define residual neuromuscular block, $64 \%, 52 \%$ and $39 \%$ of patients receiving vecuronium, atracurium or rocuronium, respectively, presented residual neuromuscular block, detectable by the inability to maintain head elevation for 5 seconds ${ }^{23}$. In a different study, the prevalence of residual neuromuscular block defined as $T_{4} / T_{1}$ ratio below 0.7 , was significantly higher in patients receiving pancuronium (26\%) as compared to those receiving vecuronium or atracurium (5.3\%). In addition, among patients receiving pancuronium and with residual neuromuscular block, $16.9 \%$ had postoperative pulmonary complications ${ }^{24}$. Diplopia or dysphasia, inability to strongly close teeth or maintain grip or head elevation for at least 5 seconds indicate residual neuromuscular block ${ }^{25}$. In these situations, quantitative neuromuscular function monitoring by acceleromyography, mechanography or electromyography, may quantify neuromuscular block level and monitor its recovery.

Approximately $15 \%$ of participants do not record postoperative clinical data. It is desirable that all anesthetic procedure steps be thoroughly recorded because this record is an invaluable piece of defense in case of legal proceedings. PACU discharge delegation to nurses and nursing technicians, which is routine for $4.05 \%$ of anesthesiologists and is allowed for selected patients by $23.61 \%$, is in disagreement with current rules, according to which PACU discharge criteria are responsibility of the anesthesiologist in charge or of the anesthesiologist on duty, to whom post-anesthetic care has been delegated. As to the need of an escort of age and responsible for ambulatory patients' discharge, $87.45 \%$ of anesthesiologists are compliant with current rules. However, anesthesiologists working in public institutions comply with this determination less frequently than those working in private institutions. Only $26.56 \%$ of participants supply written post-anesthetic recommendations to ambulatory patients. Written recommendations signed by the anesthesiologist, by the patient and/or his/her tutor in at least two copies, one of them remaining with patient's records, are the evidence that the patient and his/her tutor were informed about post-anesthetic physical and psychomotor limitations, possible complications and how to handle them, including address and contact phone numbers, thus ruling out the possibility of the physician being accused of negligence.

Revista Brasileira de Anestesiologia Vol. 53, N 4, Julho - Agosto, 2003 
This study suggests that Brazilian anesthesiologists are concerned with patients' safety in the immediate post-anesthetic period, as evidenced by the high number of well-equipped Post Anesthetic Recovery Rooms and significant percentages of isolated parameters monitoring. Some monitoring routines, primarily postoperative pain and nausea and vomiting, should be implemented according to new post-anesthetic care guidelines and new ambulatory patients discharge criteria ${ }^{26-29}$. Specific forms to record post-anesthetic parameters could be an effective way of implementing the monitoring of a larger number of post-anesthetic parameters. More attention should be given to supplying written recommendations to ambulatory patients and their tutors.

\section{AKNOWLEDGEMENTS}

To anesthesiologists and resident physicians throughout Brazil for their valuable contributions.

\section{REFERÊNCIAS - REFERENCES}

01. Cangiani LM, Porto AM - Anestesia ambulatorial. Rev Bras Anestesiol, 2000;50:68-85.

02. ASA Task Force on Postanesthetic Care: Practice guidelines for postanesthetic care. Anesthesiology, 2002;96: 742-752.

03. Backstrom $\mathrm{CH}$, Hursh-César G - Survey Research, $2^{\text {nd }}$ Ed, New York, John Wiley \& Sons, 1981;52-118.

04. Mattar FN - Pesquisa de Marketing, $5^{\mathrm{a}}$ Ed, São Paulo, Editora Atlas, 1999;300-337.

05. Eaker S, Bergström R, Bergström A et al - Response ratio to mailed epidemiologic questionnaires: a population-based randomized trial of variations in design and mailing routines. Am J Epidemiol, 1998;147:74-82.

06. DiBenedetto RJ, Graves AS, Gravenstein N et al - Pulse oximetry monitoring can change routine oxygen supplementation practices in the postanesthesia care unit. Anesth Analg, 1994; 78:365-368

07. Vegfors M, Cederholm I, Lennmarken C et al - Should oxygen be administered after laparoscopy in healthy patients? Acta Anaesthesiol Scand, 1988;32:350-352.

08. Oliveira Filho GR, Garcia JHS, Ghellar MR et al - Fatores associados com a ocorrência de hipoxemia no período pós-anestésico imediato. Rev Bras Anestesiol, 2001;51: 185-195.

09. Macario A, Weinger M, Truong P et al - Which clinical anesthesia outcomes are both common and important to avoid? The perspective of a panel of expert anesthesiologists. Anesth Analg, 1999;88:1085-1091.

10. De Witte J, Sessler DI - Perioperative shivering. Anesthesiology, 2002; $96: 467-484$.

11. Kranke $\mathrm{P}$, Eberhart $\mathrm{LH}$, Roewer $\mathrm{N}$ et al - Pharmacological treatment of postoperative shivering: a quantitative systematic review of randomized controlled trials. Anesth Analg, 2002;94: 453-460.

12. Oliveira $F^{\circ} G R$, Utzig LC, Linhares SF - Arterial blood gases during shivering and after its treatment with meperidine, oxygen or both. Br J Anaesth, 1998;80 (Suppl1):89.
13. Macario A, Dexter F - What are the most important risk factors for a patient's developing intraoperative hypothermia. Anesth Analg, 2002;94:215-220.

14. Chung F, Mezei G - Factors contributing to prolonged stay after ambulatory surgery. Anesth Analg, 1999;89: 1352-1359.

15. Sinclair DR, Chung F, Mezei G - Can postoperative nausea and vomiting be predicted. Anesthesiology, 1999;91:109-118.

16. Junger $A$, Hartmann $B$, Benson $M$ et al - The use of an anesthesia information management system for prediction of antiemetic rescue treatment at the postanesthesia care unit. Anesth Analg, 2001;92:1203-1209.

17. Pavlin DJ, Pavlin EG, Gunn HC et al - Voiding in patients managed with or without ultrasound monitoring of bladder volume after outpatient surgery. Anesth Analg, 1999;89:90-97.

18. Pavlin DJ, Pavlin EG, Fitzgibbon DR et al - Management of bladder function after outpatient surgery. Anesthesiology, 1999;91: 42-50.

19. Jin F - Should adult patients drink fluids before discharge from ambulatory surgery? Anesth Analg, 1998;87: 306-311.

20. Bengtssom M, Lüfstrüm JB, Malmqvist LA - Skin conductance responses during spinal analgesia. Acta Anaesthesiol Scand, 1985;29:67-71.

21. Brull SJ, Greene NM - Time-courses of zones of differential sensory blockade during spinal anesthesia with hyperbaric tetracaine or bupivacaine. Anesth Analg, 1989;69:342-347.

22. Pflug AE, Aasheim GM, Foster C - Sequence of return of neurological function and criteria for safe ambulation following subarachnoid block (spinal anaesthetic). Can Anaesth Soc J, 1978;25:133-139.

23. Hayes AH, Mirakhur RK, Breslin DS et al - Postoperative residual block after intermediate-acting neuromuscular blocking drugs. Anaesthesia, 2001;56:312-318.

24. Berg H, Roed J, Viby-Morgensen J et al - Residual neuromuscular block is a risk factor for postoperative pulmonary complications. A prospective, randomized, and blinded study of postoperative pulmonary complications after atracurium, vecuronium and pancuronium. Acta Anaesthesiol Scand, 1997;41:1095-1103.

25. Kopman AF, Yee PS, Neuman GG - Relationship of train-of-four fade ratio to clinical signs and symptoms of residual paralysis in awake volunteers. Anesthesiology, 1997;86:765-771.

26. Chung F, Ritchie E, Sun J - Postoperative pain in ambulatory surgery. Anesth Analg, 1997;85:808-816.

27. Chung F, Chan VWS, Ong D - A post-anesthetic discharge scoring system for home readiness after ambulatory surgery. J Clin Anesth, 1995;7:500-506.

28. Marshall SI, Chung F - Discharge criteria and complications after ambulatory surgery. Anesth Analg, 1999;88: 508-517.

29. White PF, Song D - New criteria for fast-tracking after outpatient anesthesia: a comparison with the modified Aldrete's scoring system. Anesth Analg, 1999;88: 1069-1072.

\section{RESUMEN}

Oliveira $F^{\circ}$ GR - Rutinas de Cuidados Pos-Anestésicos de Anestesiologistas Brasileños

JUSTIFICATIVA Y OBJETIVOS: No existen datos sobre la disponibilidad de equipamientos en las Salas de Recuperación Pós-Anestésica (SRPA), las rutinas de monitorización y los criterios de alta pos-anestésica utilizados por los anestesiologistas brasileños. Este estudio tuvo por finalidad obtener estas informaciones. 
MÉTODO: Fue pesquisada una muestra aleatoria de 1123 anestesiologistas. Los datos fueron analizados por tabulación simple y cruzada y por regresión logística.

RESULTADOS: Fueron analizados 271 cuestionarios $(26,59 \%)$. El estudio reveló que la mayoría de los anestesiologistas actúa en instituciones que poseen SRPA equipadas con dispositivos de reanimación, fuentes de oxígeno, aspiradores, cardioscópios, oxímetros de pulso y que mantienen anestesiologista de plantón. La existencia de monitores de función neuromuscular y dispositivos de calentamiento activo es menos frecuente. En orden decreciente de frecuencia, son monitorizados rutinariamente la presión arterial, la frecuencia cardíaca, la $\mathrm{SpO}_{2}$, el nivel de consciencia, la patencia de las vías aéreas, la frecuencia respiratoria, las náuseas y vómitos, el dolor pos-operatorio y la fuerza muscular. La oxigenioterapia, los antieméticos y el tratamiento de escalofríos pos-operatorios son administrados rutinariamente o a pacientes seleccionados por la mayoría de los anestesiologistas. La mayoría de los anestesiologistas registra los datos pos-anestésicos.

CONCLUSIONES: Este estudio sugiere que los anestesiologistas brasileños se preocupan con la seguridad de los pacientes en el período pos-anestésico inmediato, conforme lo atesta la elevada disponibilidad de equipamientos y los porcentuales expresivos de monitorización de parámetros aislados. Algunas rutinas de monitorización necesitan ser implementadas de acuerdo con las nuevas directrices de cuidados pos-anestésicos y los nuevos criterios de alta para pacientes ambulatoriales. 